decided to assess objectively the psychosocial risks to which this personnel was exposed.

Methods A research team from the scientific unit of the Institut National de Santé Publique du Québec has developed an identification grid with markers that help understanding and limiting physical and psychological risks at work. This grid includes characteristics of the workplace environment and various aspects of management practices. It is based on theoretical models such as 'Demand-LatitudeSupport' (Karasek \& Theorell, 1990), 'effort-reward imbalance' (Siegrist, 1996), 'organizational justice' (Adams, 2000), and 'prevention' (Kristensen, 1999). The assessment involved an interview guide with a scoring system of data collected from credible persons familiar with the working environment.

After adequate training in the use of the interview guide, an occupational physician and an occupational psychologist interviewed jointly each of 34 pediatricians and 3 managers and scored the 12 items of the guide according to specific recommendations. The data collected from the interviews were submitted to a thematic analysis.

Results The analysis showed that the working environment of the Department was not favorable to the return to work or work-life balance. Regarding management, the warning signals pointed to heavy workloads, lack of recognition, and communication problems. There were some protecting factors such as support from colleagues, some decision latitude (but limited possibilities of knowledge development) and, unevenly between wards, support from the hierarchy. The most negative indicators were reported by junior doctors and, as expected, by temporary personnel.

Discussion According to these results, group involving the assessment team, pediatricians, and ward managers will be formed to suggest improvements in the fields of humanresource management, communication, recognition, workloads, and occupational health.

\section{B.3 A COHORT STUDY OF SLEEP ON HEALTH PSYCHOLOGY AMONG PROFESSIONAL DRIVERS}

${ }^{1} Y$ u Jen Lin*, 1,2Yue Liang Guo, ${ }^{2}$ Saou-Hsing Liou. 'National Taiwan University, Taipei, Taiwan; ${ }^{2}$ The National Health Research Institutes, Miaoli County, Taiwan

\subsection{6/OEM-2019-EPI.169}

Background Long-term effects of sleep-related factors on risk of psychiatric disorders among professional drivers have not been conclusive. A cohort study was used to evaluate the effectiveness of subjective and objective sleep assessment tools to assess for both the 7 year risk of psychiatric disorders events. Methods: Taiwan Bus Driver Cohort Study (TBDCS) recruited 1650 professional drivers from a large bus company in Taiwan in 2005. The subjects were interviewed in person, completed the sleep assessment questionnaires (Pittsburg sleeping quality score (PSQI), Epworth daytime sleepiness score (ESS), Snore Outcomes Survey score(SOS)), and had an overnight pulse oximeter survey. Psychiatric diseases as diagnosed in the National Health Research Database were the outcomes of this study, including substance abuse, anxiety, mood, and sleep disorders. Cox proportional hazards model was performed to estimate the hazard ratio for psychiatric disorders. Results: Between 2006 and 2012, 102 bus drivers were diagnosed as having psychiatric disorders. Psychiatric disorders were related to higher PSQI score, SOS score, ODI4 levels, and ODI3 levels. The relation between PSQI and psychiatric disorders remains robust after adjusting for age, education, drinking, smoking, refreshing drinks, exercise, bus driving experience, and shift modes. Conclusion: This study showed increased psychiatric disorders in the 7 year follow-up after selfreported poor sleeping quality. Further research is warranted to develop strategies for preventing sleep-related psychiatric disorders among professional drivers.

\section{O7B.4 ARE PSYCHOSOCIAL WORKING CONDITIONS ASSOCIATED WITH SUICIDE AND INTENTIONAL SELF- HARM? A REGISTER-BASED STUDY OF 420,895 NORWEGIANS}

${ }^{1}$ Ingrid Sivesind Mehlum*, ${ }^{1}$ Therese N Hanvold, ${ }^{2}$ Lars Mehlum, ${ }^{1}$ Rachel L Hasting, ${ }^{1}$ Suzanne L Merkus, 'emerit Petter Kristensen. 'National Institute of Occupational Health (STAMI), Oslo, Norway; ${ }^{2}$ National Centre for Suicide Research and Prevention, University of Oslo, Oslo, Norway

\subsection{6/OEM-2019-EPI.170}

Objectives Poor psychosocial working conditions are associated with increased risk of mental health disorders. Some studies suggest that work factors may also increase the risk of suicidal behaviour. However, these studies mostly measured suicidal ideation, rarely completed suicides, and hardly ever used longitudinal data. We aimed to examine the relationship between selected psychosocial work factors and death by suicide, as well as hospital treatment for intentional self-harm and similar diagnoses.

Methods Data on all persons born in Norway 1967-1976, employed in $2007 \quad(n=420,895)$, were retrieved from national registers. Binary psychosocial work exposures were obtained from a job-exposure matrix for job demands, job control and leader support. Based on these, we constructed job strain (high demands, low control), and isostrain (high demands, low control, low support) exposure variables. These were linked to occupation in 2007 (ISCO88 fourdigit codes). We estimated associations (Risk Ratios, RRs) between the psychosocial work factors and four-year risk of intentional self-harm and suicide in binomial regression models, adjusted for year of birth, education level, marital history and current family pattern.

Results During 2008-2011, 884 had diagnoses of intentional self-harm (annual rate 53 per 100,000; women 56, men 49), while 164 suicided (annual rate 9.7; women 4.5, men 14.5). Intentional self-harm was associated with all work factors, except job demands and leader support among men. Associations were strongest for high isostrain, adjusted RR 1.5 (95\% confidence interval 1.2-1.7) and high job strain RR 1.3 (1.2-1.5), similar in women and men. Associations with suicide were positive only among men, with strongest associations for high job strain, RR $1.5(1.0-2.1)$.

Conclusions Psychosocial work factors were associated with intentional self-harm in both sexes, but only in men for suicide. High isostrain and high job strain showed the strongest associations. Reasons other than a causal relationship cannot be excluded, particularly health selection. 